\title{
Approximation of General Form for a Sequence of Linear Positive Operators Based on Four Parameters
}

\author{
Ali J. Mohammad ${ }^{1}$, Khalid D. Abbood ${ }^{2}$ \\ University of Basrah, College of Education for Pure Sciences, Dept. of Mathematics, Basrah, Iraq ${ }^{1,2}$ \\ alijasmoh@gmail.com¹, khalid.dhman@yahoo.com²
}

\begin{abstract}
In the present paper, we define a generalization sequence of linear positive operators based on four parameters which is reduce to many other sequences of summation-integral older type operators of any weight function (Bernstein, Baskakov, Szász or Beta). Firstly, we find a recurrence relation of the $m$-th order moment and study the convergence theorem for this generalization sequence. Secondly, we give a Voronovaskaja-type asymptotic formula for simultaneous approximation. Finally, we introduce some numerical examples to view the effect of the four parameters of this sequence.
\end{abstract}

Keywords: Sequences based on parameters, Korovkin theorem, $\boldsymbol{m}$-th order moment, Voronovaskaja -type asymptotic formula, simultaneous approximation

Date of Publication: 2018-09-30

DOI: https://doi.org/10.24297/jam.v14i2.7573

ISSN: 2347-1921

Volume: 14 Issue: 02

Journal: Journal of Advances in Mathematics

Website: https://cirworld.com

This work is licensed under a Creative Commons Attribution 4.0 International License. 


\section{Introduction}

In the past three years, many researchers have been built and studied sequences of summation-integral type operators based on parameters. These sequences give us some older sequence of summation-integral type operators, when we give suitable values for the parameters This paper is a continuation of the work of previous papers $[1,2,3,4,5,6,8$ and 11]. There are, introduce and studies general forms for sequences based on less than or equal four parameters, which are reduces to some knew sequences. Indeed, this paper is a generalization of these papers introduced in [10, 12 and 13].

The reader should be know the following sequences of linear positive operators:

- $\quad$ Summation integral Szász-Szász type sequence [10]

$$
S_{n}(f, x)=n \sum_{k=0}^{\infty} q_{n, k}(x) \int_{0}^{\infty} q_{n, k}(t) f(t) d t, n \in N, \quad x \in[0, \infty)
$$

where

$$
q_{n, k}(x)=\frac{(n x)^{k}}{k !} e^{-n x}
$$

- $\quad$ Summation integral Baskakov-Szász type sequence [12]

$$
V_{n}(f, x)=n \sum_{k=0}^{\infty} p_{n, k}(x) \int_{0}^{\infty} q_{n, k}(t) f(t) d t
$$

where

$$
p_{n, k}(x)=\left(\begin{array}{c}
n+k-1 \\
k
\end{array}\right) x^{k}(1+x)^{-n-k} .
$$

- $\quad$ Summation integral Beta-Szász type sequence [13]

$$
M_{n}(f, x)=\sum_{k=0}^{\infty} \beta_{n, k}(x) \int_{0}^{\infty} q_{n, k}(t) f(t) d t, x \in[0, \infty),
$$

where

$$
\beta_{n, k}(x)=\frac{1}{\mathrm{~B}(k+1, n)} x^{k}(1+x)^{-n-k-1},
$$

$\mathrm{B}(k+1, n)$ being the Beta function given by $\frac{\Gamma(k+1) \Gamma(n)}{\Gamma(n+k+1)}$.

Suppose that $C[0, \infty)$ denotes the space of all continuous real-valued functions on the interval $[0, \infty)$ the subspace $C_{\gamma}[0, \infty)$ of the space $C[0, \infty)$ is defined as:

$C_{\gamma}[0, \infty):=\left\{f \in C[0, \infty):|f(t)|=O\left(e^{\gamma t}\right)\right.$, for some $\left.\gamma>0\right\}$.

The space $C_{\gamma}[0, \infty)$ in normed by the norm: $\|f\|_{C_{\gamma}}=\sup _{t \in[0, \infty)} \frac{|f(t)|}{e^{\gamma t}}, f \in C_{\gamma}[0, \infty)$.

We define and study our sequence based on four parameters $\rho>0, c \in N^{0} \cup\{-1\}$ and $r \in N^{0}=N \cup\{0\}$ as follows: 


$$
H_{\alpha}^{\rho}(f, x, c, r)=\sum_{k=0}^{\infty} p_{\alpha, k}(x, c, r) \int_{0}^{\infty} \theta_{\alpha, k}^{\rho}(t) f(t) d t, x \in[0, \infty),
$$

where $p_{\alpha, k}(x, c, r)=\frac{(-x)^{k}}{k !} \phi_{\alpha, c, r}^{(k)}(x)$,

$$
\phi_{\alpha, c, r}(x)=\left\{\begin{array}{cc}
e^{-\alpha x}, & c=r=0 \\
(1+c x)^{-\frac{\alpha}{c}-\frac{r}{c},} & \text { otherwise }
\end{array}\right.
$$

and

$$
\theta_{\alpha, k}^{\rho}(t)=\frac{\alpha \rho}{\Gamma(k \rho+1)} e^{-\alpha \rho t}(\alpha \rho t)^{k \rho} .
$$

Sometime, we write the operators $H_{\alpha}^{\rho}(f, x, c, r)$ as $H_{\alpha}^{\rho}(f, x, c, r)=\int_{0}^{\infty} W_{\alpha}^{\rho}(t, x) f(t) d t$, where $W_{\alpha}^{\rho}(t, x)$ is called the kernel of the operators $H_{\alpha}^{\rho}(f, x, c, r)$ and define as:

$$
W_{\alpha}^{\rho}(t ; x)=\sum_{k=0}^{\infty} p_{\alpha, k}(x, c, r) \theta_{\alpha, k}^{\rho}(t)
$$

where $\delta(t)$ being the Dirac-delta function.

Our new sequence is give us many other older sequences when $\alpha=n \in N$ for examples

- $\quad H_{n}^{1}(f, x, 0,0)=S_{n}(f, x)$;

- $\quad H_{n}^{1}(f, x, 0,1)=V_{n}(f, x)$;

- $\quad H_{n}^{1}(f, x, 1,1)=M_{n}(f, x)$.

In addition, from our sequence, we can get summation integral Bernstein-Szász type sequence. Here, we refer to the sequence getting by putting $\alpha=n, \rho=1, c=-1$ and $r=0$, i.e.

$$
H_{n}^{1}(f, x,-1,0)=B_{n}(f, x)=n \sum_{k=0}^{n} b_{n, k}(x) \int_{0}^{\infty} q_{n, k}(t) f(t) d t,
$$

where

$$
b_{n, k}(x)=\left(\begin{array}{l}
n \\
k
\end{array}\right) x^{k}(1-x)^{n-k}, x \in[0,1]
$$

During this paper, we assume that $M$ is a positive real constant not necessarily the same in different cases.

\section{Preliminary Results}

First, we need to introduce some properties of the classical following sequence:

$$
Y_{\alpha}(f, x, c, r)=\sum_{k=0}^{\infty} p_{\alpha, k}(x, c, r) f\left(\frac{k}{\alpha}\right), x \in[0, \infty)
$$

where $f \in C_{\gamma}[0, \infty)$ and $m \in N^{0}$.

The $m$-th order moment of the sequence $Y_{\alpha}(f, x, c, r)$ is defined as

$$
\mu_{\alpha, m}(x)=Y_{\alpha}\left((t-x)^{m} ; x, c, r\right)=\sum_{k=0}^{\infty} p_{\alpha, k}(x, c, r)\left(\frac{k}{\alpha}-x\right)^{m} .
$$


Lemma 2.1. The function $\mu_{\alpha, m}(x)$ defined above has the folloing properties:

$\mu_{\alpha, 0}(x)=1, \mu_{\alpha, 1}(x)=\frac{r x}{\alpha}, \mu_{\alpha, 2}(x)=\frac{x(1+c x)}{\alpha}+\frac{r x(1+c x)}{\alpha^{2}}+\frac{r^{2} x^{2}}{\alpha^{2}}$ and has the following recurrence relation

$$
\alpha \mu_{\alpha, m+1}(x)=x(1+c x)\left[\mu_{\alpha, m}^{\prime}(x)+m \mu_{\alpha, m-1}(x)\right]+r x \mu_{\alpha, m}(x), m \geq 1 .
$$

Further, the following consequences of $\mu_{\alpha, m}(x)$ are hold:

(i) $\quad \mu_{\alpha, m}(x)$ is polynomial in $x$ of degree at most $m$;

(i) For every $x \in[0, \infty), \mu_{\alpha, m}(x)=O\left(\alpha^{-\left[\frac{m+1}{2}\right]}\right)$.

Proof: It is clear that the relation is true at $x=0$. Now, for $x \in[0, \infty)$, we have:

$$
\begin{aligned}
& \mu_{\alpha, m}(x)=\sum_{k=0}^{\infty} p_{\alpha, k}(x, c, r)\left(\frac{k}{\alpha}-x\right)^{m} \\
& \mu_{\alpha, m}^{\prime}(x)=\sum_{k=0}^{\infty} p_{\alpha, k}^{\prime}(x, c, r)\left(\frac{k}{\alpha}-x\right)^{m}-m \sum_{k=0}^{\infty} p_{\alpha, k}(x, c, r)\left(\frac{k}{\alpha}-x\right)^{m-1} \\
& x(1+c x) \mu_{\alpha, m}^{\prime}(x)=-m x(1+c x) \mu_{\alpha, m-1}(x)+\sum_{k=0}^{\infty} p_{\alpha, k}(x, c, r)\left(\frac{k}{\alpha}-x\right)^{m}[k-(\alpha+r) x] \\
& x(1+c x)\left[\mu_{\alpha, m}^{\prime}(x)+m \mu_{\alpha, m-1}(x)\right]=\alpha \sum_{k=0}^{\infty} p_{\alpha, k}(x, c, r)\left(\frac{k}{\alpha}-x\right)^{m+1}-r x \sum_{k=0}^{\infty} p_{\alpha, k}(x, c, r)\left(\frac{k}{\alpha}-x\right)^{m} .
\end{aligned}
$$

Form which (2.1) is immediate. Form the values of $\mu_{\alpha, 0}(x), \mu_{\alpha, 1}(x)$, using the indication on $m$ and the recurrence relation above, we can easily prove that $\mu_{\alpha, m}(x)=O\left(\alpha^{-\left[\frac{m+1}{2}\right]}\right)$.

Lemma 2.2. For $H_{\alpha}^{\rho}\left(t^{m} ; x, c, r\right)$ and $m \in N^{0}$ the following conditions are hold

$H_{\alpha}^{\rho}(1 ; x, c, r)=1$

$H_{\alpha}^{\rho}(t ; x, c, r)=x+\frac{r x}{\alpha}+\frac{1}{(\alpha \rho)}$

$H_{\alpha}^{\rho}\left(t^{2} ; x, c, r\right)=x^{2}+\frac{x(1+c x)}{\alpha}+\frac{r x(1+c x)}{\alpha^{2}}+\frac{3 x}{\alpha \rho}+\frac{3 r x}{\alpha^{2} \rho}+\frac{r^{2} x^{2}}{\alpha^{2}}+\frac{2 r x^{2}}{\alpha}+\frac{2}{(\alpha \rho)^{2}}$.

Hence, by applying Korovkin theorem [7] for $H_{\alpha}^{\rho}(f ; x, c, r)$, we have that

$$
H_{\alpha}^{\rho}(f(t), x, c, r) \rightarrow f(x) \text { as } \alpha \rightarrow \infty .
$$

In the same manner, we define the $m$-th order moment $T_{n, m}(x)$ for the sequence $H_{\alpha}^{\rho}(. ; x, c, r)$ by:

$$
T_{\alpha, m}(x)=H_{\alpha}^{\rho}\left((t-x)^{m} ; x, c, r\right)=\sum_{k=0}^{\infty} p_{\alpha, k}(x, c, r) \int_{0}^{\infty} \theta_{\alpha, k}^{\rho}(t)(t-x)^{m} d t .
$$


Lemma 2.3. For the function $T_{\alpha, m}(x)$, we have:

$T_{\alpha, 0}(x)=1 ;$

$T_{\alpha, 1}(x)=\frac{r x}{\alpha}+\frac{1}{\alpha \rho}$

$T_{\alpha, 2}(x)=\frac{x(1+c x)}{\alpha}+\frac{r x(1+c x)}{\alpha^{2}}+\frac{r^{2} x^{2}}{\alpha^{2}}+\frac{x}{\alpha \rho}+\frac{3 r x}{\alpha^{2} \rho}+\frac{2}{(\alpha \rho)^{2}}$

also, we have the following recurrence relation

$\alpha T_{\alpha, m+1}(x)$

$=x(1+c x) T_{\alpha, m}^{\prime}(x)+m x\left[\frac{1}{\rho}+(1+c x)\right] T_{\alpha, m-1}(x)+\left[\frac{m+1}{\rho}+r x\right] T_{\alpha, m}(x)$.

In addition, the function $T_{\alpha, m}(x)$ is a polynomial in $x$ of degree at most $m$ and $T_{\alpha, m}(x)=O\left(\alpha^{-\left[\frac{m+1}{2}\right]}\right)$, where $\left[\frac{m+1}{2}\right]$ denotes the integer part of $\frac{m+1}{2}$.

Proof: By direct computation, the values $T_{\alpha, 0}(x), T_{\alpha, 1}(x)$ and $T_{\alpha, 2}(x)$ can be easily follow, we prove (2.2). For $x=0$ it clearly holds. For $x \in(0, \infty)$, we have:

Next,

$T_{\alpha, \mathrm{m}}^{\prime}(x)=\sum_{k=0}^{\infty}{p^{\prime}}_{\alpha, k}(x, c, r) \int_{0}^{\infty} \theta_{\alpha, k}^{\rho}(t)(t-x)^{m} d t-m \sum_{k=0}^{\infty} p_{\alpha, k}(x, c, r) \int_{0}^{\infty} \theta_{\alpha, k}^{\rho}(t)(t-x)^{m-1} d t$.

Using the equation $x(1+c x) p_{\alpha, k}^{\prime}(x, c, r)=(k-(\alpha+r) x) p_{\alpha, k}(x, c, r)$, we get

$x(1+c x) T_{\alpha, m}^{\prime}(x)=\sum_{k=0}^{\infty} p_{\alpha, k}(x, c, r)(k-(\alpha+r) x) \int_{0}^{\infty} \theta_{\alpha, k}^{\rho}(t)(t-x)^{m} d t-m x(1+c x) T_{\alpha, m-1}(x)$.

Let $k-(\alpha+r) x=\left(k-\alpha t+\frac{1}{\rho}\right)-\frac{1}{\rho}+\alpha(t-x)-r x$.

Since $\frac{1}{\rho} \frac{d}{d t}\left(t \theta_{\alpha, k}^{\rho}(t)\right)=\left(k-\alpha t+\frac{1}{\rho}\right) \theta_{\alpha, k}^{\rho}(t)$.

$x(1+c x) T_{\alpha, m}^{\prime}(x)$

$=\sum_{k=0}^{\infty} p_{\alpha, k}(x, c, r) \int_{0}^{\infty} \frac{1}{\rho} \frac{d}{d t}\left(t \theta_{\alpha, k}^{\rho}(t)\right)(t-x)^{m} d t-\frac{1}{\rho} \sum_{k=0}^{\infty} p_{\alpha, k}(x, c, r) \int_{0}^{\infty} \theta_{\alpha, k}^{\rho}(t)(t-x)^{m} d t+\alpha T_{\alpha, m+1}(x)$

$-r x T_{\alpha, m}(x)-m x(1+c x) T_{\alpha, m-1}(x)$.

Since

$$
\int_{0}^{\infty} \frac{d}{d t}\left(t \theta_{\alpha, k}^{\rho}(t)\right)(t-x)^{m} d t=-m \int_{0}^{\infty} t \theta_{\alpha, k}^{\rho}(t)(t-x)^{m-1} d t
$$

The identity $t=(t-x)+x$ 


$$
\begin{gathered}
x(1+c x) T_{\alpha, m}^{\prime}(x)=\frac{-m}{\rho} \sum_{k=0}^{\infty} p_{\alpha, k}(x, c, r) \int_{0}^{\infty} \theta_{\alpha, k}^{\rho}(t)(t-x)^{m} d t-\frac{m x}{\rho} \sum_{k=0}^{\infty} p_{\alpha, k}(x, c, r) \int_{0}^{\infty} \theta_{\alpha, k}^{\rho}(t)(t-x)^{m-1} d t \\
-\frac{1}{\rho} \sum_{k=0}^{\infty} p_{\alpha, k}(x, c, r) \int_{0}^{\infty} \theta_{\alpha, k}^{\rho}(t)(t-x)^{m} d t-r x T_{\alpha, m}(x)-m x(1+c x) T_{\alpha, m-1}(x)+\alpha T_{\alpha, m+1}(x) .
\end{gathered}
$$

Finally, we get the recurrence relation state above.

From which (2.2) is immediate.

From the values of $T_{\alpha, 0}(x), T_{\alpha, 1}(x)$, using the induction on $m$ and the recurrence relation above, we can easily prove that $T_{\alpha, m}(x)=O\left(\alpha^{-\left[\frac{m+1}{2}\right]}\right)$ for every $x \in[0, \infty)$.

Lemma 2.4. Let $\gamma$ and $\delta$ be any two positive real number and $[a, b] \subset[0, \infty]$ be any bounded interval. Then, for any $m>0$ there exists a constant $M$ depending on $m$ only and $\alpha$ independent of $M$.

We have

$$
\left\|\int_{|t-x| \geq \delta} W_{\alpha}(t, x) e^{\gamma t} d t\right\|_{C[a, b]}=O\left(\alpha^{-m}\right),
$$

where $\|.\|_{C[a, b]}$ means the sup norm in the space $C[a, b]$.

Proof: Using the Schwartz inequality for integration and then for summation, the proof this lemma is easily follows and the details are omitted.

Lemma 2.5. For every $x \in(0, \infty)$ ands $\in N^{0}$, there exist polynomials $Q_{i, j, r}(x, c, r)$ in $x$ independent of $\alpha$ and $k$

$$
(x(1+c x))^{s} p_{\alpha, k}^{(s)}(x, c, r)=\sum_{\substack{2 i+j \leq s \\ i, j \geq 0}} \alpha^{i}(k-(\alpha+r) x)^{j} Q_{i, j, r}(x, c, r) p_{\alpha, k}(x, c, r) .
$$

Proof: For the cases $c=1$ and $r=0$ the proof of this relation can be seen in [9].

\section{Main Results}

In this section, we introduce a Voronovskaja type asymptotic formula for the sequence $H_{\alpha}^{\rho}(f ; x, c, r)$, we show that the $s$-th derivative $\left.\frac{d^{s}}{d w^{s}} H_{\alpha}^{\rho}(f ; w, c, r)\right|_{w=x}$ is an approximation process for $f^{(s)}(x), s=1,2, \ldots$.

Theorem 3.1. For $\gamma>0, f \in C_{\gamma}[0, \infty)$ and $f^{(s)}$ exists at a point $x \in(0, \infty)$, then we have

$$
\left.\lim _{\alpha \rightarrow \infty} \frac{d^{s}}{d w^{s}} H_{\alpha}^{\rho}(f ; w, c, r)\right|_{w=x}=f^{(s)}(x)
$$

Further, if $f^{(s)}$ exists and is continuous on $(a-\eta, b+\eta) \subset(0, \infty), \eta>0$, then (3.1) holds uniformly in $[a, b]$.

Proof: by Taylor's expansion, we have

$$
f(t)=\sum_{i=0}^{s} \frac{f^{(i)}(x)}{i !}(t-x)^{i}+\psi(t, x)(t-x)^{s}, t \in[0, \infty)
$$


where $\psi(t, x) \rightarrow 0$ as $t \rightarrow x$ hence,

$$
\begin{gathered}
\left.\frac{d^{s}}{d w^{s}} H_{\alpha}^{\rho}(f(t) ; w, c, r)\right|_{w=x}=\left.\sum_{i=0}^{s} \frac{f^{(i)}(x)}{i !} \frac{d^{s}}{d w^{s}} H_{\alpha}^{\rho}\left((t-x)^{i} ; w, c, r\right)\right|_{w=x}+\left.\frac{d^{s}}{d w^{s}} H_{\alpha}^{\rho}\left(\psi(t, x)(t-x)^{s} ; w, c, r\right)\right|_{w=x} \\
:=I_{1}+I_{2} .
\end{gathered}
$$

$$
\begin{aligned}
& I_{1}=\left.\frac{f^{(s)}(x)}{s !} \frac{d^{s}}{d w^{s}} H_{\alpha}^{\rho}\left((t-x)^{s} ; w, c, r\right)\right|_{w=x} \\
& =\left.\frac{f^{(s)}(x)}{s !} \frac{d^{s}}{d w^{s}} H_{\alpha}^{\rho}\left(\sum_{j=0}^{s}\left(\begin{array}{l}
s \\
j
\end{array}\right) t^{j}(-x)^{s-j} ; w, c, r\right)\right|_{w=x} \\
& =\left.\frac{f^{(s)}(x)}{s !} \frac{d^{s}}{d w^{s}} H_{\alpha}^{\rho}\left(t^{s} ; w, c, r\right)\right|_{w=x}
\end{aligned}
$$

Since

$$
H_{\alpha}^{\rho}\left(t^{s} ; x, c, r\right)=\frac{\prod_{i=0}^{s-1}(\alpha+r+c i)}{(\alpha \rho)^{s}}(\rho x)^{s}+\left(\frac{s(s-1) \rho}{2}+\frac{s(s+1)}{2}\right) \frac{\prod_{i=0}^{s-2}(\alpha+r+c i)}{(\alpha \rho)^{s}}(\rho x)^{s-1}+O\left(\alpha^{-2}\right) .
$$

Then

$$
\frac{d^{s}}{d x^{s}} H_{\alpha}^{\rho}\left(t^{s} ; x, c, r\right)=\frac{\prod_{i=0}^{s-1}(\alpha+r+c i)}{(\alpha)^{s}} s !
$$

Therefore $I_{1}=f^{(s)}(x)$ as $\alpha \rightarrow \infty$

$$
\begin{aligned}
& I_{2}=\left.\frac{d^{s}}{d w^{s}} H_{\alpha}^{\rho}\left(\psi(t, x)(t-x)^{s} ; w, c, r\right)\right|_{w=x} \\
& =\left.\frac{d^{s}}{d w^{s}} \sum_{k=0}^{\infty} p_{\alpha, k}(x, c, r) \int_{0}^{\infty} \theta_{\alpha, k}^{\rho}(t) \psi(t, x)(t-x)^{s} d t\right|_{w=x} .
\end{aligned}
$$

From Lemma (2.5)

$$
\begin{array}{r}
(x(1+c x))^{s} p_{\alpha, k}^{(s)}(x, c, r)=\sum_{\substack{2 i+j \leq s \\
i, j \geq 0}} \alpha^{i}(k-(\alpha+r) x)^{j} Q_{i, j, s}(x, c, r) p_{\alpha, k}(x, c, r) \\
\leq \sum_{\substack{2 i+j \leq s \\
i, j \geq 0}} \frac{\left|Q_{i, j, s}(x, c, r)\right|}{(x(1+c x))^{s}} \alpha^{i} \sum_{k=0}^{\infty} p_{\alpha, k}(x, c, r)|k-(\alpha+r) x|^{j} \int_{0}^{\infty} \theta_{\alpha, k}^{\rho}(t)|\psi(t, x)||t-x|^{s} d t
\end{array}
$$

Since $\psi(t, x) \rightarrow 0$ as $\rightarrow x$, then for a given $\epsilon>0$ there exists $\delta>0$ such that $|\psi(t, x)|<\varepsilon$ whenever $|t-x|<\delta$. For $|t-x| \geq \delta$, we have $|\psi(t, x)(t-x)| \leq M e^{\gamma t}$, for some $M>0$. Thus,

$$
\begin{gathered}
I_{3}=M \sum_{\substack{2 i+j \leq s \\
i, j \geq 0}} \alpha^{i} \sum_{k=0}^{\infty} p_{\alpha, k}(x, c, r)|k-(\alpha+r) x|^{j}\left\{\varepsilon \int_{|t-x|<\delta} \theta_{\alpha, k}^{\rho}(t)|t-x|^{s} d t+\int_{|t-x| \geq \delta}^{\infty} \theta_{\alpha, k}^{\rho}(t) e^{\gamma t} d t\right\} \\
:=I_{4}+I_{5},
\end{gathered}
$$

7927 
where $M=\operatorname{Sup}_{\substack{2 i+j \leq s \\ i, j \geq 0}} \frac{\left|Q_{i, j, s}(x, c, r)\right|}{(x(1+c x))^{s}}$.

Now, applying Schwarz inequality for integration, summation, w conclude

$I_{4}=\varepsilon M \sum_{\substack{2 i+j \leq s \\ i, j \geq 0}} \alpha^{i} \sum_{k=0}^{\infty} p_{\alpha, k}(x, c, r)|k-(\alpha+r) x|^{j}\left(\int_{0}^{\infty} \theta_{\alpha, k}^{\rho}(t)|t-x|^{2 s} d t\right)^{\frac{1}{2}}\left(\int_{0}^{\infty} \theta_{\alpha, k}^{\rho}(t) d t\right)^{\frac{1}{2}}$

Since $\int_{0}^{\infty} \theta_{\alpha, k}^{\rho}(t) d t=1$

$\leq \varepsilon M \sum_{\substack{2 i+j \leq s \\ i, j \geq 0}} \alpha^{i}\left(\sum_{k=0}^{\infty} p_{\alpha, k}(x, c, r)|k-(\alpha+r) x|^{2 j}\right)^{\frac{1}{2}}\left(\sum_{k=0}^{\infty} p_{\alpha, k}(x, c, r) \int_{0}^{\infty} \theta_{\alpha, k}^{\rho}(t)|t-x|^{2 s} d t\right)^{\frac{1}{2}}$

$\leq \varepsilon M O\left(\alpha^{-\frac{s}{2}}\right) \sum_{\substack{2 i+j \leq s \\ i, j \geq 0}} \alpha^{i} O\left(\alpha^{-\frac{j}{2}}\right)=\varepsilon O(1)$.

Since $\varepsilon>0$ is arbitrary then $I_{4} \rightarrow 0 \quad$ as $\alpha \rightarrow \infty$.

$$
\begin{aligned}
& I_{5}=M \sum_{\substack{2 i+j \leq s \\
i, j \geq 0}} \alpha^{i} \sum_{k=0}^{\infty} p_{\alpha, k}(x, c, r)|k-(\alpha+r) x|^{j} \int_{|t-x| \geq \delta} \theta_{\alpha, k}^{\rho}(t) e^{\gamma t} d t \\
& \leq M \sum_{\substack{2 i+j \leq s \\
i, j \geq 0}} \alpha^{i} \sum_{k=0}^{\infty} p_{\alpha, k}(x, c, r)|k-(\alpha+r) x|^{j}\left(\int_{|t-x| \geq \delta} \theta_{\alpha, k}^{\rho}(t)(t-x)^{2 \gamma} d t\right)^{\frac{1}{2}}\left(\int_{|t-x| \geq \delta} \theta_{\alpha, k}^{\rho}(t) d t\right)^{\frac{1}{2}} \\
& \leq M \sum_{\substack{2 i+j \leq s \\
i, j \geq 0}} \alpha^{i}\left(\sum_{k=0}^{\infty} p_{\alpha, k}(x, c)|k-(\alpha+r) x|^{2 j}\right)^{\frac{1}{2}}\left(\sum_{k=0}^{\infty} p_{\alpha, k}(x, c) \int_{|t-x| \geq \delta} \theta_{\alpha, k}^{\rho}(t)(t-x)^{2 \gamma} d t\right)^{\frac{1}{2}} \\
& =\sum_{\substack{2 i+j \leq r \\
i, j \geq 0}} \alpha^{i} O\left(\alpha^{\frac{2 i+j}{2}}\right) O\left(\alpha^{\frac{-s}{2}}\right)=O(1) \\
& I_{5} \rightarrow 0 \text { as } \alpha \rightarrow \infty .
\end{aligned}
$$

Hence, $I_{2}=O(1)$ as $\alpha \rightarrow \infty$.

Combining the estimates of $I_{1}$ and $I_{2}$ we obtain (3.1).

Theorem 3.2. Let $f \in C_{\gamma}[0, \infty)$ For some $\gamma>0$. if $f$ admits a derivative of order $(s+2)$ at a fixed point, $x \in$ $(0, \infty)$, then we have

$$
\begin{aligned}
\lim _{\alpha \rightarrow \infty} \alpha\left(\frac{d^{s}}{d w^{s}} H_{\alpha}^{\rho}(f ; w, c, r)-\left.f^{s}(x)\right|_{w=x}\right) & =\left(\frac{s(s-1) c}{2}+s r\right) f^{(s)}(x) \\
& +\left(x(r+c s)+\frac{s(\rho+1)}{2 \rho}+\frac{1}{\rho}\right) f^{(s+1)}(x)+\left(\frac{x}{2 \rho}[(\rho+1)+c \rho x]\right) f^{(s+2)}(x) .
\end{aligned}
$$


Further, if $f^{(s+2)}$ exists and is continuous on $(a-\eta, b+\eta) \subset(0, \infty), \eta>0$, then (3.2) holds uniformly in $[a, b]$.

Proof: By Taylor's expansion, we have

$$
f(t)=\sum_{i=0}^{s+2} \frac{f^{(i)}(x)}{i !}(t-x)^{i}+\psi(t, x)(t-x)^{s+2} .
$$

Where $\psi(t, x) \rightarrow 0$ as $t \rightarrow x$ hence,

$$
\begin{aligned}
& \left.\frac{d^{s}}{d w^{s}} H_{\alpha}^{\rho}(f(t) ; w, c, r)\right|_{w=x}=\left.\sum_{i=0}^{s+2} \frac{f^{(i)}(x)}{i !} \frac{d^{s}}{d w^{s}} H_{\alpha}^{\rho}\left((t-x)^{i} ; w, c, r\right)\right|_{w=x}+\left.\frac{d^{s}}{d w^{s}} H_{\alpha}^{\rho}\left(\psi(t, x)(t-x)^{s+2} ; w, c, r\right)\right|_{w=x} \\
& \lim _{\alpha \rightarrow \infty} \alpha\left(\frac{d^{s}}{d w^{s}} H_{\alpha}^{\rho}(f ; w, c, r)-\left.f^{(s)}(x)\right|_{w=x}\right)=\lim _{\alpha \rightarrow \infty} \alpha\left(\sum_{i=0}^{s+2} \frac{f^{(i)}(x)}{i !}\left(\frac{d^{s}}{d w^{s}} H_{\alpha}^{\rho}\left((t-x)^{i} ; w, c, r\right)\right)_{w=x}-f^{(s)}(x)\right) \\
& +\lim _{\alpha \rightarrow \infty} \alpha\left(\left.\frac{d^{s}}{d w^{s}} H_{\alpha}^{\rho}\left(\psi(t, x)(t-x)^{s+2} ; w, c, r\right)\right|_{w=x}\right) \\
& :=I_{1}+I_{2} \text {. } \\
& I_{1}=\alpha\left\{\left.\frac{f^{(s)}(x)}{s !} \frac{d^{s}}{d w^{s}} H_{\alpha}^{\rho}\left(t^{s} ; w, c, r\right)\right|_{w=x}+\frac{f^{(s+1)}(x)}{(s+1) !}\left[\left.(s+1)(-x) \frac{d^{s}}{d w^{s}} H_{\alpha}^{\rho}\left(t^{s} ; w, c, r\right)\right|_{w=x}\right.\right. \\
& \left.+\left.\frac{d^{s}}{d w^{s}} H_{\alpha}^{\rho}\left(t^{s+1} ; w, c\right)\right|_{w=x}\right]+\frac{f^{(s+2)}(x)}{(s+2) !}\left[\left.\frac{(s+1)(s+2)}{2} x^{2} \frac{d^{s}}{d w^{s}} H_{\alpha}^{\rho}\left(t^{s} ; w, c, r\right)\right|_{w=x}\right. \\
& \left.\left.+\left.(s+2)(-x) \frac{d^{s}}{d w^{s}} H_{\alpha}^{\rho}\left(t^{s+1} ; w, c, r\right)\right|_{w=x}+\left.\frac{d^{s}}{d w^{s}} H_{\alpha}^{\rho}\left(t^{s+2} ; w, c, r\right)\right|_{w=x}\right]-f^{(s)}(x)\right\} \\
& =\alpha f^{(s)}(x)\left[\frac{\prod_{i=0}^{s-1}(\alpha+r+c i)}{(\alpha)^{s}}-1\right]+\alpha \frac{f^{(s+1)}(x)}{(s+1) !}\left[(s+1) !(-x) \frac{\prod_{i=0}^{s-1}(\alpha+r+c i)}{(\alpha)^{s}}\right. \\
& \left.+\frac{\prod_{i=0}^{s}(\alpha+r+c i)}{(\alpha)^{s+1}}(s+1) ! x+(s+1) !\left(\frac{s}{2} \rho+\frac{(s+2)}{2}\right) \frac{\prod_{i=0}^{s-1}(\alpha+r+c i)}{\rho(\alpha)^{s+1}}\right] \\
& +\alpha \frac{f^{(s+2)}(x)}{(s+2) !}\left[\frac{(s+2) !}{2} \frac{\prod_{i=0}^{s-1}(\alpha+r+c i)}{(\alpha)^{s}} x^{2}+(s+2) ! \frac{\prod_{i=0}^{s}(\alpha+r+c i)}{(\alpha)^{s+1}}\left(-x^{2}\right)\right. \\
& +\frac{(s+2) !}{2} \frac{\prod_{i=0}^{s+1}(\alpha+r+c i)}{(\alpha)^{s+2}} x^{2}+(s+2) !\left(\frac{s}{2} \rho+\frac{(s+2)}{2}\right) \frac{\prod_{i=0}^{s-1}(\alpha+r+c i)}{\rho(\alpha)^{s+1}}(-x) \\
& \left.+\left(\frac{(s+1)}{2} \rho+\frac{(s+3)}{2}\right)(s+2) ! \frac{\prod_{i=0}^{s}(\alpha+r+c i)}{\rho(\alpha)^{s+2}} x\right] \\
& :=J_{1}+J_{2}+J_{3} \text {. } \\
& J_{1}=\left(\frac{s(s-1) c}{2}+s r\right) f^{(s)}(x) ; \\
& J_{2}=f^{(s+1)}(x)\left(x(r+c s)+\frac{s(\rho+1)}{2 \rho}+\frac{1}{\rho}\right) ;
\end{aligned}
$$




$$
J_{3}=f^{(s+2)}(x)\left(\frac{x}{2 \rho}[(\rho+1)+c \rho x]\right) .
$$

Since $I_{2} \rightarrow 0$ as $\alpha \rightarrow \infty$.

Thus, we obtain (3.2).

\section{Numerical Examples}

In this section, we introduce some numerical examples for the sequence $H_{\alpha}^{\rho}(f(t) ; x, c, r)$ by taking two test functions and show the approximation by this sequence $H_{\alpha}^{\rho}(f(t) ; x, c, r)$ and its derivatives to the function being approximate. The results are explain by graphs and the error functions occur between the test functions and the approximations.

Suppose that $g$ is an integrable function on interval $[a, b]$ and $h$ is an approximate to the function $g$ in the interval $[a, b]$. We define the error $E$ as follows:

$$
E=\int_{a}^{b}|g(t)-h(t)| d t .
$$

Example 4.1. Put $\alpha=20$ and $\rho=1,2,5$ for the test function $f(x)=\sqrt{\frac{1}{4}-\left(x-\frac{1}{2}\right)^{2}}, x \in[0.1]$, we get the figures (4.1)-(4.3) respectively.
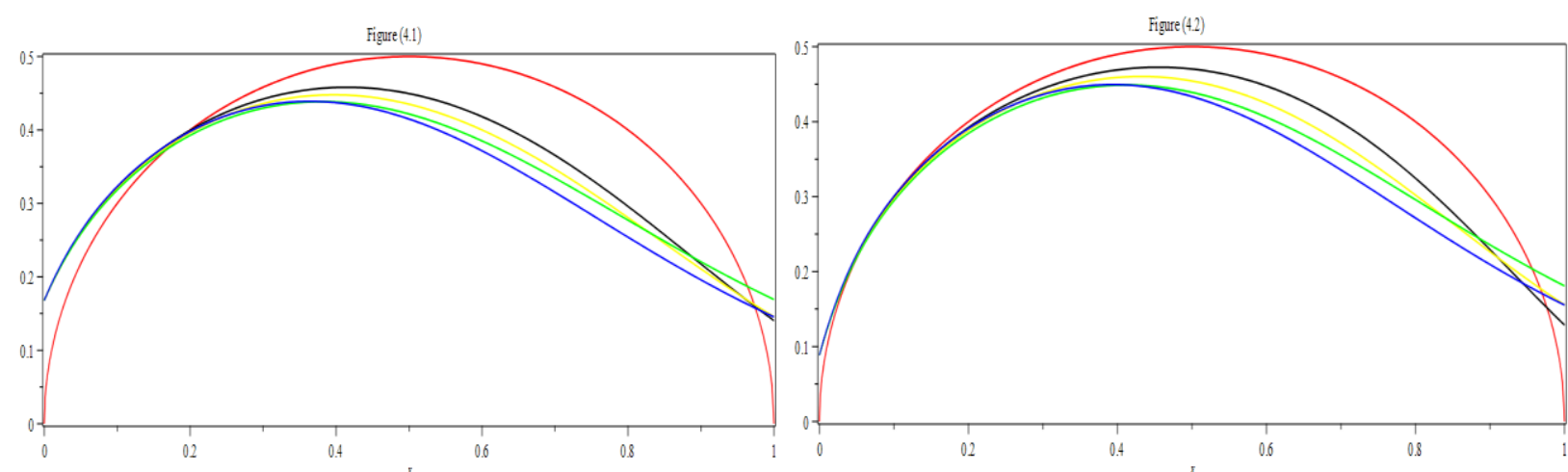

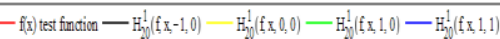

- $f(x)$ est findion $-H_{20}^{2}(f x,-1,0)-H_{20}^{2}(f, 0,0)-H_{20}^{2}(f, x, 0)-H_{20}^{2}(f, 1,1)$

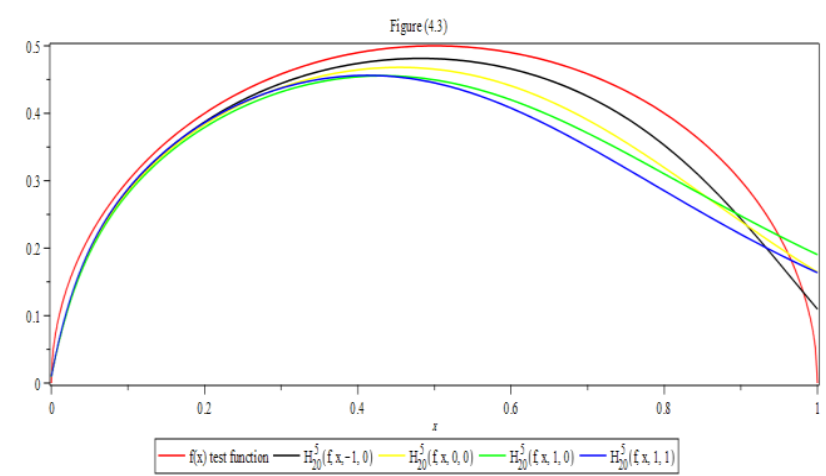


Example 4.2. Put $\alpha=40,80,120$ and $\rho=1$ for the test function $f(x)=\sqrt{\frac{1}{4}-\left(x-\frac{1}{2}\right)^{2}}, x \in[0.1]$, we get the figures (4.4)-(4.6) respectively.
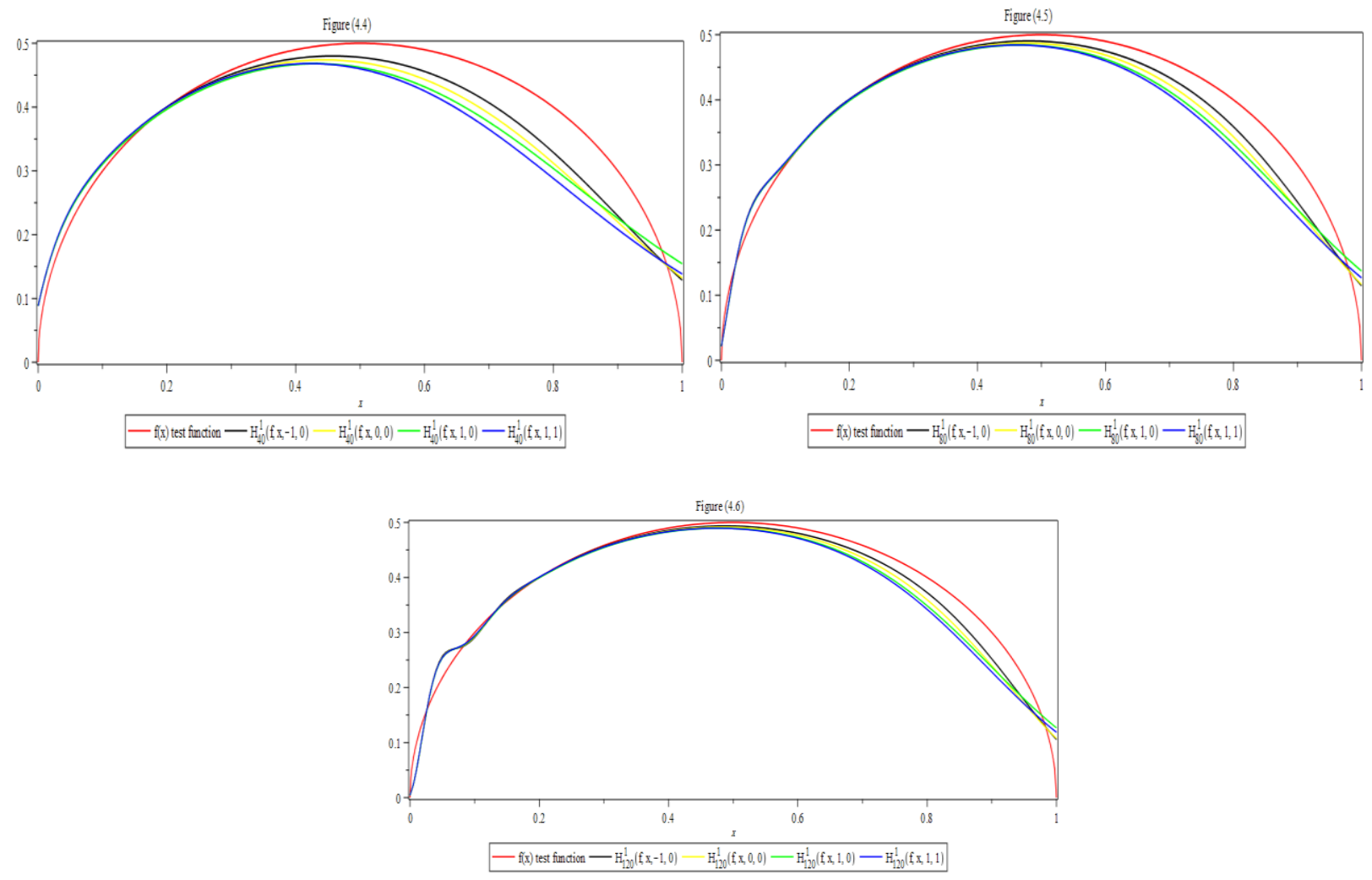

Example 4.3. Put $\alpha=20$ and $\rho=1,2,5$ for the test function $f(x)=\sin (10 x) e^{-3 x}, x \in[0,1]$, we get the figures (4.7)-(4.9) respectively.
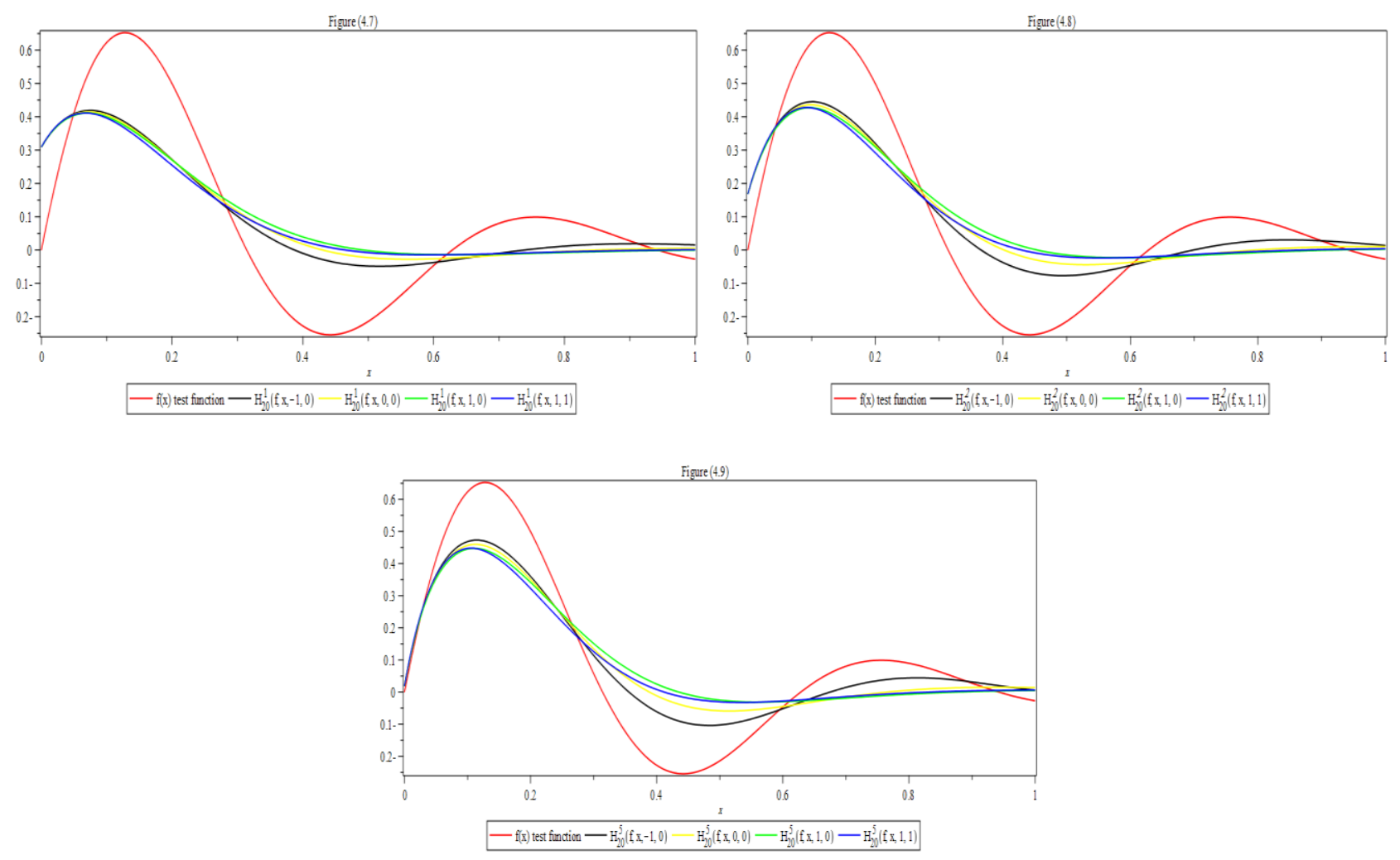
Example 4.4. Put $\alpha=40,80,120$ and $\rho=1$ for the test function $f(x)=\sin (10 x) e^{-3 x}, x \in[0,1]$, we get the figures (4.10)-(4.12) respectively.
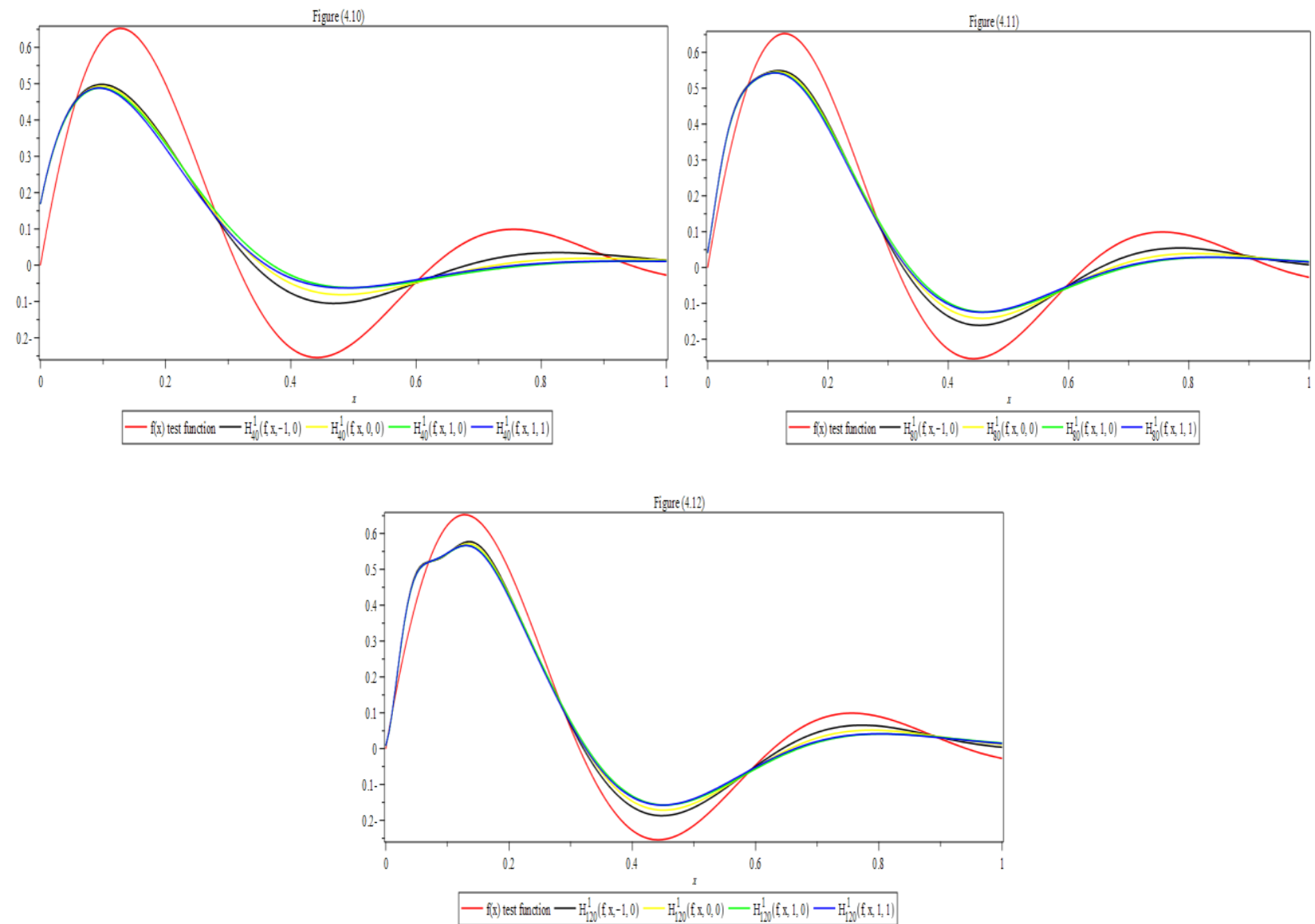

Exempla 4.5. Put $\alpha=20,40$ and $\rho=1$ the convergence of the sequence $\frac{d}{d x} H_{\alpha}^{\rho}(f(t) ; x, c, r)$ to the function $\frac{d}{d x} f(x)=\frac{d}{d x}\left(\sqrt{\frac{1}{4}-\left(x-\frac{1}{2}\right)^{2}}\right), x \in[0,1]$, we get the figures (4.13)-(4.14) respectively.
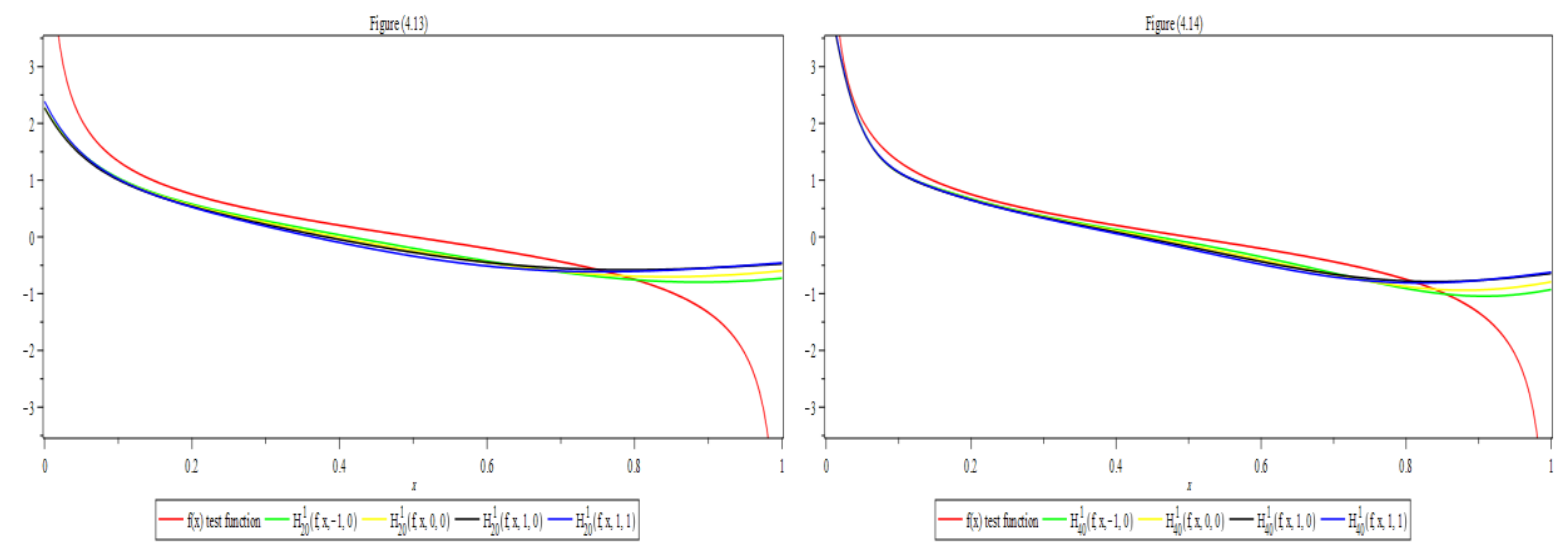
Exempla 4.6. Put $\alpha=20,40$ and $\rho=1$ the convergence of the sequence $\frac{d}{d x} H_{\alpha}^{\rho}(f(t) ; x, c, r)$ to the function $\frac{d}{d x} f(x)=\frac{d}{d x}\left(\sin (10 x) e^{-3 x}\right), x \in[0,1]$, we get the figures (4.15)-(4.16) respectively.
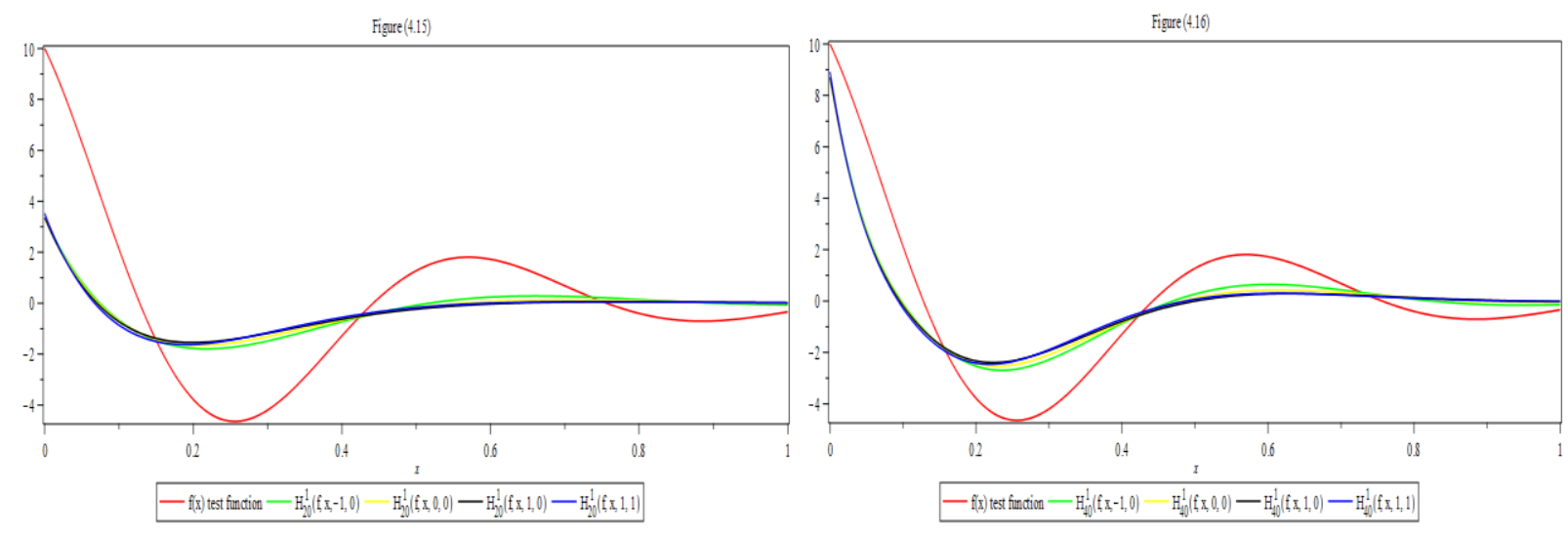

Table 4.1. Shows the value of the error function $E$ by the parameter values $\alpha, \rho$ of the sequence $H_{\alpha}^{\rho}(f, x, c, r)$ for function the test $f(x)=\sqrt{\frac{1}{4}-\left(x-\frac{1}{2}\right)^{2}}$

\begin{tabular}{|c|c|c|c|}
\hline The sequence & $\rho=1$ & $\rho=1.5$ & $\rho=2$ \\
\hline$H_{20}^{\rho}(f, x,-1,0)$ & $0.5231988685 e-1$ & $0.4035461651 e-1$ & $0.3401310448 e-1$ \\
\hline$H_{40}^{\rho}(f, x,-1,0)$ & $0.3098022066 e-1$ & $0.2340296322 e-1$ & $0.2027696454 e-1$ \\
\hline$H_{40.5}^{\rho}(f, x,-1,0)$ & $0.3037282140 e-1$ & $0.2305826975 e-1$ & $0.2010000244 e-1$ \\
\hline$H_{20}^{\rho}(f, x, 0,0)$ & $0.6127126920 e-1$ & $0.5073029289 e-1$ & $0.4553608828 e-1$ \\
\hline$H_{40}^{\rho}(f, x, 0,0)$ & $0.3803138880 e-1$ & $0.3128938169 e-1$ & $0.2874132397 e-1$ \\
\hline$H_{40.5}^{\rho}(f, x, 0,0)$ & $0.3819753878 e-1$ & $0.3148135068 e-1$ & $0.2893827328 e-1$ \\
\hline$H_{20}^{\rho}(f, x, 1,0)$ & $0.6658951445 e-1$ & $0.5729171730 e-1$ & $0.5299131396 e-1$ \\
\hline$H_{40}^{\rho}(f, x, 1,0)$ & $0.4250067617 e-1$ & $0.3640006110 e-1$ & $0.3426809680 e-1$ \\
\hline$H_{40.5}^{\rho}(f, x, 1,0)$ & $0.4260499198 e-1$ & $0.3652658173 e-1$ & $0.3439444871 e-1$ \\
\hline$H_{20}^{\rho}(f, x, 1,1)$ & $0.7520779764 e-1$ & $0.6518004854 e-1$ & $0.5992990033 e-1$ \\
\hline$H_{40}^{\rho}(f, x, 1,1)$ & $0.4762326366 e-1$ & $0.4109374653 e-1$ & $0.3853042539 e-1$ \\
\hline$H_{40.5}^{\rho}(f, x, 1,1)$ & $0.4776705635 e-1$ & $0.4125428055 e-1$ & $0.3870234942 e-1$ \\
\hline
\end{tabular}

Table (4.1) 
Table 4.2. Shows the value of the error function by parameter values $\alpha, \rho$ for the sequence $H_{\alpha}^{\rho}(f, x, c, r)$ for function the test $f(x)=\sin (10 x) e^{-3 x}$

\begin{tabular}{|c|c|c|c|}
\hline The sequence & $\rho=1$ & $\rho=1.5$ & $\rho=2$ \\
\hline$H_{20}^{\rho}(f, x,-1,0)$ & 0.1153700137 & 0.1026977270 & $0.9480519059 e-1$ \\
\hline$H_{40}^{\rho}(f, x,-1,0)$ & $0.7965284146 e-1$ & $0.06704181936 e-1$ & $0.5991964902 e-1$ \\
\hline$H_{40.5}^{\rho}(f, x,-1,0)$ & $0.7888248106 e-1$ & $0.6635425644 e-1$ & $0.5929919364 e-1$ \\
\hline$H_{20}^{\rho}(f, x, 0,0)$ & 0.1248684215 & 0.1156266940 & 0.1101446950 \\
\hline$H_{40}^{\rho}(f, x, 0,0)$ & $0.9010708858 e-1$ & $0.08001907225 e-1$ & $0.7452265499 e-1$ \\
\hline$H_{40.5}^{\rho}(f, x, 0,0)$ & $0.8948387285 e-1$ & $0.7941388577 e-1$ & $0.7393553518 e-1$ \\
\hline$H_{20}^{\rho}(f, x, 1,0)$ & 0.1311079457 & 0.1238737934 & 0.1197078019 \\
\hline$H_{40}^{\rho}(f, x, 1,0)$ & $0.9809666512 e-1$ & $0.8957011436 e-1$ & $0.8501446977 e-1$ \\
\hline$H_{40.5}^{\rho}(f, x, 1,0)$ & $0.9749668073 e-1$ & $0.8897284530 e-1$ & $0.8442355702 e-1$ \\
\hline$H_{20}^{\rho}(f, x, 1,1)$ & 0.1300450161 & 0.1223008671 & 0.1177245063 \\
\hline$H_{40}^{\rho}(f, x, 1,1)$ & $0.9742135996 e-1$ & $0.8859542122 e-1$ & $0.8381471380 e-1$ \\
\hline$H_{40.5}^{\rho}(f, x, 1,1)$ & $0.9683332206 e-1$ & $0.8801244668 e-1$ & $0.8324007680 e-1$ \\
\hline
\end{tabular}

Table (4.2)

Table 4.3. Shows the value of the error function $E$ by parameter values $\alpha, \rho$ for the sequence $\frac{d}{d x} H_{\alpha}^{\rho}(f, x, c, r)$ for function the test $\frac{d}{d x} f(x)=\frac{d}{d x}\left(\sqrt{\frac{1}{4}-\left(x-\frac{1}{2}\right)^{2}}\right)$

\begin{tabular}{|c|c|c|}
\hline The sequence & $\rho=1$ & $\rho=1.5$ \\
\hline$\frac{d}{d x} H_{20}^{\rho}(f, x,-1,0)$ & 0.5165158746 & 0.4315109183 \\
\hline$\frac{d}{d x} H_{40}^{\rho}(f, x,-1,0)$ & 0.3698853954 & 0.3196723100 \\
\hline$\frac{d}{d x} H_{20}^{\rho}(f, x, 0,0)$ & 0.5535021159 & 0.4855199331 \\
\hline$\frac{d}{d x} H_{40}^{\rho}(f, x, 0,0)$ & 0.4017649664 & 0.3643542686 \\
\hline$\frac{d}{d x} H_{20}^{\rho}(f, x, 1,0)$ & 0.5909387065 & 0.5263493438 \\
\hline$\frac{d}{d x} H_{40}^{\rho}(f, x, 1,0)$ & 0.4358381024 & 0.3984571765 \\
\hline
\end{tabular}




\begin{tabular}{|c|c|c|}
\hline$\frac{d}{d x} H_{20}^{\rho}(f, x, 1,1)$ & 0.6107287290 & 0.5468538012 \\
\hline$\frac{d}{d x} H_{40}^{\rho}(f, x, 1,1)$ & 0.4515302033 & 0.4174223054 \\
\hline
\end{tabular}

Table (4.3)

Table 4.4. Shows the value of the error function $E$ by parameter values $\alpha, \rho$ for the sequence $\frac{d}{d x} H_{\alpha}^{\rho}(f, x, c, r)$ for function the test $\frac{d}{d x} f(x)=\frac{d}{d x}\left(\sin (10 x) e^{-3 x}\right)$

\begin{tabular}{|c|c|c|}
\hline The sequence & 1 & 1.5 \\
\hline$\frac{d}{d x} H_{20}^{\rho}(f, x,-1,0)$ & 1.558174800 & 1.357430849 \\
\hline$\frac{d}{d x} H_{40}^{\rho}(f, x,-1,0)$ & 1.045151546 & 0.8463874786 \\
\hline$\frac{d}{d x} H_{20}^{\rho}(f, x, 0,0)$ & 1.637468279 & 1.474332363 \\
\hline$\frac{d}{d x} H_{40}^{\rho}(f, x, 0,0)$ & 1.151186273 & 0.9814928752 \\
\hline$\frac{d}{d x} H_{20}^{\rho}(f, x, 1,0)$ & 1.687781683 & 1.543273588 \\
\hline$\frac{d}{d x} H_{40}^{\rho}(f, x, 1,0)$ & 1.227638145 & 1.074604477 \\
\hline$\frac{d}{d x} H_{20}^{\rho}(f, x, 1,1)$ & 1.684164816 & 1.535270668 \\
\hline$\frac{d}{d x} H_{40}^{\rho}(f, x, 1,1)$ & 1.222612969 & \\
\hline
\end{tabular}

Table (4.4)

\section{Conclusions}

The numerical results show the effect of parameter $\rho, c$ and $r$ values in the approximation by our sequence. It turns out that,

- The approximation increases whenever $\rho$ increase

- The best approximation occur in our examples when $c=-1$ and $r=0$

- When $\alpha$ is sufficiently large the effect of the parameters $\rho, c$ and $r$ is vanish 


\section{References}

[1] A.J. Mohammad and A. K. Hassan, A generalization of Baskakov operators of summation-integral- Phillips type form, Basrah J. of Sci. vol. 31(1), 28-40, (2013).

[2] D. Soybas, Approximation with modified Phillips operators, J. Nonlinear Sci. Appl., 10, 5803-5812 (2017).

[3] E.E. Berdysheva, Operators of Durrmeyer type with respect to arbitrary measure, Springer Proceeding in Mathematics \& Statistics, Roorkee, India, Dec. (2014).

[4] H.M. Srivastava and V. Gupta, A certain family of summation-integral type operators. Math. Comput. Model.37, 1307-1315 (2003).

[5] M. Goyal and P.N. Agrawal, Degree of approximation by certain genuine hybrid operators, Springer Proceeding in Mathematics \& Statistics, Roorkee, India, Dec. (2014).

[6] M. Heilmann and I. Rasa, $\mathrm{k}$ th order Kantorovich modification of linking Baskakov-type operators, Springer Proceeding in Mathematics \& Statistics, Roorkee, India, Dec. (2014).

[7] P.P. Korovkin: Linear Operators and Approximation Theory, Hindustan publ. Corp. Delhi, 1960 (Translated from Russian Edition) (1959).

[8] R. Păltănea, Modified Szász-Mirakjan operators of integral form, Carpathian J. Math. 24 (2008), 378-385.

[9] R.P. Sinha, P.N. Agrawal and V. Gupta, On simultaneous approximation by modified Baskakov operators, Bull. Soc. Math. Belg. 43, 217-231 (1991).

[10] S.M. Mazhar and V. Totik, Approximation by modified Szász operators, Acra Sci. Math,

49, 257-269 (1985).

[11] V. Cupta, M. Rassisas, P.N. Agrawal and M. Goyal, Approximation with certain genuine hybrid operators, Serbia N. of Sci (2017).

[12] V. Gupta and G.S. Srivastava, Simultaneous approximation by Baskakov-Szász type operators, Bull. Soc. math, tome 37(85), nr 3-4 (1993).

[13] V. Gupta and S. Srivastava, Convergence of derivatives by summation-integral type operators, math, India, (1995). 